\title{
Les apports en lipides d'origine animale de la population française : résultats de l'étude INCA2
}

Lionel LAFAY

Éric VERGER

Observatoire des consommations alimentaires
—épidémiologie nutritionnelle,
PASER/DERNS/Afssa,
27-31, avenue du Cénéral-Leclerc,
94701 Maisons-Alfort cedex,
France
<I.lafay@afssa.fr>

Si les apports en lipides totaux et ceux en acides gras saturés (AGS), mono-insaturés (AGMI), poly-insaturés (AGPI) de la population sont relativement bien documentés (Afssa, 2009 ; Astorg et al., 2004 ; Féart et al., 2007 ; Razanamahefa et al., 2005), l'origine de ces apports selon leur source (animale ou végétale) l'est moins.

Àl'aide des données recueillies dans le cadre de la seconde étude individuelle nationale des consommations alimentaires (étude INCA2), il a été possible d'évaluer pour chaque participant la quantité de lipides consommés en tenant compte de son origine (animale ou végétale), après notamment décomposition des plats composés et des aliments complexes en ingrédients. Ce travail n'avait pas pu être réalisé à l'aide des données de la première étude nationale des consommations alimentaires (étude INCA1), mais un travail préliminaire $d^{\prime}$ 'identification des aliments sources de lipides $d^{\prime}$ origine animale et d'origine mixte (sans pouvoir précisément identifier la part de chaque origine) avait été cependant réalisé.

Les objectifs du présent travail sont, d'une part, de présenter les apports en lipides d'origine animale dans la population française ainsi que leurs vecteurs en 2006-2007 et, d'autre part, de présenter l'évolution des apports de lipides d'origines animale et mixte entre 1998-1999 et 2006-2007.

\section{Population et méthodologie}

\section{Population}

L'étude INCA2 s'est déroulée en trois vagues entre fin 2005 et avril 2007 afin de tenir compte

\begin{abstract}
Using food intake data recorded by 2,624 adults (18-79 yo) and 1,455 children (3-17 yo), crossed with the nutritional food composition table (CIQUAL), animal origin fat intake was estimated. Mean total fat intake varies between $69 \mathrm{~g} / \mathrm{d}$ in girls and $100 \mathrm{~g} / \mathrm{d}$ in men. Animal origin fat represents, on average, between $62 \%$ in women and $66 \%$ in men of total fat intake. Animal origin fats are provided by milk and milk products for more than $60 \%$ and by meat for less than a third. Animal-and mixed origin fat decreased by $14 \%$ between INCA1 and INCA2. During the same period animal origin products (meat, milk, cheeses, etc.) consumption decreased in both children and adults. However a part of these evolutions can result from methodological differences as the best identification of added fat used for cooking or the improvements in the food identification and in the food composition table.
\end{abstract}

Key words: animal origin fat, French population, INCA2 study

des variations saisonnières (Afssa, 2009). Elle a inclus 2624 adultes (18-79 ans) et 1455 enfants (3-17 ans).

La sélection des participants a été réalisée selon un plan de sondage à trois degrés, stratifié selon le degré d'urbanisation et la région: tirage d'unités primaires (UP) au premier degré, puis de logements au deuxième degré, puis d'un individu au sein du logement pour le troisième degré. La base de sondage utilisée pour tirer les logements est le recensement général de la population de 1999 (RP99) de I'Institut national de la statistique et des études économiques (Insee), complété par les bases BSLN (base de sondage des logements neufs) et SITADEL (système d'information et de traitement automatisé des données élémentaires sur les logements et les locaux) pour les logements construits après 1999. La méthodologie du plan de sondage et le tirage des fiches adresses ont été réalisés par I'Insee.

\section{Méthodes}

Deux populations distinctes ont été incluses dans l'étude : les enfants de 3 à 17 ans et les adultes de 18 à 79 ans. Le recrutement des participants par les enquêteurs a été effectué par téléphone (si le numéro du logement tiré au sort a pu être trouvé) ou en face-à-face. II a consisté à entrer en contact avec le foyer, à expliquer l'étude, à établir la composition du foyer et à effectuer le tirage au sort de la personne participante. Une fois l'accord de participation recueilli, I'enquêteur effectuait deux visites au domicile des participants : la première pour déposer l'ensemble des documents à remplir et à expliquer avec précision la manière de les remplir ; la seconde pour récupérer ces documents, en contrôler le remplissage et poser un questionnaire informatisé. Par ailleurs, des appels de soutien ou de relance étaient effectués entre les deux visites pour s'assurer du bon déroulement de l'étude.

Le recueil des consommations alimentaires a été réalisé avec un carnet de consommation de sept jours consécutifs. Chaque journée était décomposée en trois repas et trois prises inter-repas. Pour chaque prise ou repas, le participant devait décrire le détail de tous les aliments et boissons consommés, estimer la quantité consommée à l'aide d'un manuel de photographies de portions ou de mesures ménagères ou encore de grammages ou de volumes unitaires et indiquer les informations sur le type de produit (industriel/fait maison, frais/conserve/surgelé, enrichi/allégé/ou non). En plus des données de consommations alimentaires, l'étude a également recueilli au moyen d'un questionnaire autoadministré des informations qualitatives relatives à l'utilisation de matières grasses pour différentes préparations (cuisson des viandes, des poissons, assaisonnement des féculents, des légumes, assaisonnement des crudités, etc.) qui ont été répercutées dans les carnets de consommation pour les repas pris au domicile, lorsque la description des plats ne permettait pas de le savoir.

Les informations recueillies sur les carnets de consommation alimentaire ont été vérifiées et harmonisées par des diététiciennes.

La codification des aliments a reposé sur une nomenclature créée spécifiquement pour 
l'étude, comportant 1280 items classés en 43 groupes et compatible avec celle de la composition nutritionnelle des aliments du Centre d'information sur la qualité des aliments (Ciqual) de l'Afssa (Afssa, 2008).

Une table de recettes décomposant les aliments composés ou complexes en ingrédients de la nomenclature a été réalisée. Elle comporte 617 aliments qui sont décomposés en 1241 ingrédients qui disposent donc tous d'un vecteur nutritionnel. Pour chaque aliment ou ingrédient a été attribuée une origine : animale ou végétale.

Les apports nutritionnels moyens ont ainsi pu être obtenus en croisant les données de consommation avec les données de composition nutritionnelle de la table du Ciqual. Les apports en lipides d'origine animale ont ainsi été évalués pour chaque individu. Le ratio entre apports lipidiques d'origine animale et lipides totaux a permis de définir pour chaque individu la part des lipides d'origine animale dans sa ration lipidique quotidienne. L'origine animale a, par ailleurs, été décomposée en 14 contributeurs majeurs : volaille, porc, mouton, veau, bœuf, lapin, œufs, beurre, lait, fromages, crème fraîche, autres produits laitiers, produits de la mer et autres.

Lors de l'étude INCA1, les aliments vecteurs de lipides d'origine animale ou mixte avaient été identifiés en distinguant dix sources majeures : volaille, porc, veau, mouton, bœuf, lapin et autres viandes, œufs, lait et dérivés, produits de la mer et autres. Cette classification a également été appliquée aux données de l'étude INCA2 afin d'étudier l'évolution des apports en lipides d'origine animale et mixte.

Afin de se rapprocher le plus possible de la répartition de la population française, un redressement par calage sur marges a été effectué sur les deux sous-échantillons « adultes » et « enfants » en tenant compte de la région, de la taille d'agglomération, de la taille du ménage, du sexe, de l'âge, de la profession et de la catégorie sociale du chef de ménage et de la saison d'enquête.

Les données sont présentées indépendamment chez les adultes (18-79 ans) et les enfants (3-17 ans), globalement et par sexe. Les apports en lipides d'origine animale, bruts et relatifs (apports lipidiques d'origine animale/ apports lipidiques totaux) sont également présentés par classes d'âge. Les analyses ont été conduites sous SAS 9.1 à l'aide de la procédure surveymeans afin de prendre en compte le plan de sondage de l'étude.

Les participants adultes de l'étude INCA2 ( $n=706)$ qui ont, volontairement ou non, sous-estimé leurs apports caloriques ont été identifiés selon le critère de Goldberg (1991) et adapté par Black (2000) et exclus des analyses portant sur les consommations alimentaires et les apports nutritionnels. Les enfants $(n=11)$ dont les apports étaient également trop bas au regard de la distribution par âge et par sexe ont été également exclus.

\section{Résultats}

Le tableau 1 présente les apports en calories avec et sans alcool, lipides, protides, glucides, alcool et en AGS, AGMl et AGPI chez les adultes et les enfants. Les adultes consomment en moyenne $2066 \mathrm{kcal} / \mathrm{j}$, hors alcool, apport

Tableau 1. Apports en calories, en lipides, en protides, en glucides, en acides gras saturés, mono-insaturés et poly-insaturés chez les adultes (18-79 ans) et les enfants (3-17 ans) de l'étude INCA2 (2006-2007) - moyenne \pm écart-type.

\begin{tabular}{|c|c|c|c|c|c|c|}
\hline & \multicolumn{3}{|l|}{ Adultes } & \multicolumn{3}{|l|}{ Enfants } \\
\hline & Hommes & Femmes & Ensemble & Garçons & Filles & Ensemble \\
\hline AESA (kcal/j) & $2348 \pm 582$ & $1809 \pm 416^{* * *}$ & $2066 \pm 558$ & $1897 \pm 537$ & $1635 \pm 405^{* * *}$ & $1770 \pm 490$ \\
\hline AET (kcal/j) & $2500 \pm 598$ & $1855 \pm 426^{* * *}$ & $2162 \pm 596$ & $1904 \pm 540$ & $1641 \pm 407^{* * *}$ & $1777 \pm 492$ \\
\hline Lipides ( $\mathrm{g} / \mathrm{j})$ & $100 \pm 28$ & $80 \pm 21^{\star \star *}$ & $89 \pm 26$ & $80 \pm 25$ & $69 \pm 18^{\star * *}$ & $75 \pm 22$ \\
\hline Lipides (\% AESA) & $38,5 \pm 6,1$ & $39,6 \pm 5,3^{* *}$ & $39,1 \pm 5,7$ & $38,0 \pm 4,9$ & $38,1 \pm 4,9$ & $38,0 \pm 4,9$ \\
\hline AGMI (g/j) & $35,7 \pm 11,0$ & $28,6 \pm 8,5^{\star \star *}$ & $32,0 \pm 10,2$ & $28,3 \pm 9,7$ & $24,2 \pm 7,1^{* * *}$ & $26,3 \pm 8,7$ \\
\hline AGMI (\% AGT) & $39,1 \pm 5,2$ & $39,2 \pm 5,0$ & $39,2 \pm 5,0$ & $38,6 \pm 4,2$ & $38,3 \pm 4,2$ & $38,4 \pm 4,2$ \\
\hline AGPI (g/j) & $14,5 \pm 6,3$ & $12,3 \pm 5,0^{* * *}$ & $13,4 \pm 5,7$ & $10,7 \pm 5,0$ & $9,5 \pm 4,1^{* * *}$ & $10,1 \pm 4,6$ \\
\hline AGPI (\% AGT) & $16,0 \pm 5,8$ & $16,9 \pm 5,0^{* *}$ & $16,5 \pm 5,3$ & $14,6 \pm 4,9$ & $14,9 \pm 4,4$ & $14,7 \pm 4,6$ \\
\hline $\operatorname{AGS}(\mathrm{g} / \mathrm{j})$ & $41,2 \pm 14,3$ & $32,1 \pm 9,9^{* * *}$ & $36,4 \pm 12,7$ & $34,2 \pm 11,3$ & $29,6 \pm 8,3^{* * *}$ & $32,0 \pm 10,1$ \\
\hline AGS (\% AGT) & $44,8 \pm 6,8$ & $44,0 \pm 6,0^{*}$ & $44,4 \pm 6,4$ & $46,7 \pm 5,5$ & $46,9 \pm 5,5$ & $46,8 \pm 5,5$ \\
\hline Protides $(\mathrm{g} / \mathrm{j})$ & $100 \pm 26$ & $74 \pm 17^{* * *}$ & $86 \pm 25$ & $73 \pm 22$ & $62 \pm 16^{* * *}$ & $68 \pm 20$ \\
\hline Protides (\% AESA) & $17,2 \pm 2,9$ & $16,7 \pm 2,8^{* * *}$ & $16,9 \pm 2,8$ & $15,6 \pm 2,5$ & $15,3 \pm 2,3$ & $15,4 \pm 2,4$ \\
\hline Glucides $^{\mathrm{a}}$ (g/j) & $262 \pm 85$ & $199 \pm 57^{* * *}$ & $229 \pm 76$ & $221 \pm 71$ & $192 \pm 58^{* * *}$ & $207 \pm 66$ \\
\hline Glucides $^{\mathrm{a}}(\% \mathrm{AESA})$ & $44,3 \pm 7,1$ & $43,8 \pm 5,9$ & $44,0 \pm 6,4$ & $46,5 \pm 5,5$ & $46,7 \pm 5,5$ & $46,6 \pm 5,5$ \\
\hline Alcool (g/j) & $21,0 \pm 22,4$ & $5,7 \pm 8,1^{* * *}$ & $13,0 \pm 17,3$ & $0,3 \pm 2,6$ & $0,2 \pm 1,4$ & $0,3 \pm 2,0$ \\
\hline Alcool (\% AET) & $5,9 \pm 6,2$ & $2,1 \pm 2,9^{* * *}$ & $3,9 \pm 4,9$ & $0,1 \pm 0,8$ & $0,1 \pm 0,5$ & $0,1 \pm 0,7$ \\
\hline
\end{tabular}

AESA : apport énergétique sans alcool ; AET : apport énergétique total ; AGMI : acides gras mono-insaturés ; AGPI : acides gras poly-insaturés ; AGS : acides gras saturés ; $A G T$ : acides gras totaux (ACS + AGMI + AGPI).

Test des différences entre hommes et femmes / entre filles et garçons : ${ }^{*} p<0,05 ;{ }^{* *} p<0,01 ;{ }^{* * *} p<0,001$.

${ }^{a}$ Glucides disponibles (amidon + glucides simples + polyols). 
plus élevé chez les hommes que chez les femmes d'environ $30 \%$. Les enfants ont un apport calorique moyen proche de $1770 \mathrm{kcal} / \mathrm{j}$, avec un apport majoré de $16 \%$ chez les garçons par rapport aux filles. Les hommes consomment davantage de lipides (100 vs $80 \mathrm{~g} / \mathrm{j})$ et les garçons que les filles ( 80 vs $69 \mathrm{~g} / \mathrm{j})$. La contribution des lipides aux apports caloriques est cependant plus élevée chez les femmes $(39,6 \%)$ que chez les hommes $(38,5 \%)$ et ne diffère pas entre les filles et les garçons (38,0\%). Les adultes consomment en moyenne $32,0 \mathrm{~g} / \mathrm{j}$ d'AGMI, 13,4 g/j d'AGPI et 36,4 g/j d'AGS. Ces valeurs sont significativement plus élevées chez les hommes que chez les femmes. Chez les femmes, la part des AGPI au sein des acides gras totaux est significativement plus élevée alors que celle des AGMI est identique et celle des AGS moins élevée que chez les hommes. Chez les enfants, les apports bruts des trois types $d^{\prime}$ acides gras sont significativement plus élevés chez les garçons, mais les apports relatifs sont similaires. Par rapport aux adultes, la part des AGS est globalement plus élevée chez les enfants alors que celles des AGMl et surtout des AGPI sont plus basses.

Tableau 2. Apports en lipides d'origine animale en grammes par jour ( $g / j)$ et en pourcentage (\%) de la ration lipidique totale par sexe et par classe d'âge chez les participants à l'étude INCA2 (2006-2007).

\begin{tabular}{|c|c|c|c|c|}
\hline & & Garçons & Filles & Ensemble \\
\hline \multirow[t]{2}{*}{$3-10$ ans } & $\mathrm{g} / \mathrm{j}$ & $47,6 \pm 14,9$ & $42,7 \pm 12,3^{* * *}$ & $45,3 \pm 14,0$ \\
\hline & $\%$ & $64,4 \pm 11,0$ & $64,0 \pm 11,4$ & $64,2 \pm 11,2$ \\
\hline \multirow[t]{2}{*}{$11-14$ ans } & $\mathrm{g} / \mathrm{j}$ & $54,6 \pm 16,1$ & $46,1 \pm 16,0^{* * *}$ & $50,4 \pm 16,6$ \\
\hline & $\%$ & $64,7 \pm 11,0$ & $64,1 \pm 10,9$ & $64,0 \pm 11,1$ \\
\hline \multirow[t]{2}{*}{$15-17$ ans } & $\mathrm{g} / \mathrm{j}$ & $56,8 \pm 23,3$ & $40,4 \pm 14,3^{* * *}$ & $48,4 \pm 20,8$ \\
\hline & $\%$ & $63,1 \pm 12,1$ & $59,4 \pm 13,8^{* *}$ & $61,2 \pm 13,1$ \\
\hline \multirow[t]{3}{*}{ Ensemble } & $\mathrm{g} / \mathrm{j}$ & $51,2 \pm 17,6^{c}$ & $43,1 \pm 14,0^{* * *}, \mathrm{~b}$ & $47,3 \pm 16,5^{c}$ \\
\hline & $\%$ & $64,2 \pm 11,2$ & $62,8 \pm 12,0^{*}, c$ & $63,5 \pm 11,6^{c}$ \\
\hline & & Hommes & Femmes & Ensemble \\
\hline \multirow[t]{2}{*}{$18-34$ ans } & $\mathrm{g} / \mathrm{j}$ & $67,8 \pm 19,7$ & $49,0 \pm 17,4^{\star * *}$ & $56,5 \pm 20,5$ \\
\hline & $\%$ & $65,6 \pm 10,8$ & $61,2 \pm 12,3^{* * *}$ & $62,9 \pm 11,9$ \\
\hline \multirow[t]{2}{*}{$35-54$ ans } & $\mathrm{g} / \mathrm{j}$ & $67,4 \pm 24,8$ & $49,7 \pm 17,3^{* * *}$ & $57,7 \pm 22,8$ \\
\hline & $\%$ & $65,2 \pm 12,8$ & $62,0 \pm 12,2^{* *}$ & $63,5 \pm 12,6$ \\
\hline \multirow[t]{2}{*}{$55-79$ ans } & $\mathrm{g} / \mathrm{j}$ & $61,4 \pm 20,6$ & $46,7 \pm 16,6^{* * *}$ & $54,9 \pm 20,3$ \\
\hline & $\%$ & $63,9 \pm 13,4$ & $60,1 \pm 14,9^{* *}$ & $62,2 \pm 14,2$ \\
\hline \multirow[t]{2}{*}{ Ensemble } & $\mathrm{g} / \mathrm{j}$ & $65,0 \pm 22,2^{c}$ & $48,6 \pm 17,2^{* * * a}$ & $56,4 \pm 21,4^{a}$ \\
\hline & $\%$ & $64,8 \pm 12,6$ & $61,2 \pm 13,1^{* * *}$ & $62,9 \pm 13,0$ \\
\hline
\end{tabular}

Test des différences entre hommes et femmes / entre filles et garçons : ${ }^{*} p<0,05 ;{ }^{* *} p<0,01 ;{ }^{* * *} p<0,001$. Test des différences entre les classes d'âge : ${ }^{a} p<0,05 ;{ }^{b} p<0,01 ;{ }^{c} p<0,001$.
Les quantités brutes de lipides animaux et leur contribution aux lipides totaux sont présentées par sexe et par classes d'âges dans le tableau 2. Chez l'enfant, l'apport en lipides d'origine animale est en moyenne proche de $47,3 \mathrm{~g} / \mathrm{j}$, soit $63,5 \%$ de l'apport lipidique total. Cet apport est plus élevé chez les garçons que chez les filles d'environ $8 \mathrm{~g} / \mathrm{j}$. L'apport brut en lipides animaux augmente avec l'âge mais de manière plus marquée chez les garçons. La part des lipides animaux au sein des lipides totaux reste stable chez les garçons (64\% en moyenne), alors qu'elle tend à diminuer avec l'âge chez les filles (de 64,0\% chez les 3 à 10 ans à $59,4 \%$ chez les 15 à 17 ans). Ainsi, la part des lipides animaux au sein des lipides totaux, similaire entre garçons et filles jusqu'à 14 ans, devient ensuite significativement supérieure chez les garçons.

Chez l'adulte, l'apport en lipides d'origine animale est en moyenne proche de $56 \mathrm{~g} / \mathrm{j}$, soit $63 \%$ de I'apport lipidique total. Quelle que soit la tranche d'âge, les apports bruts et relatifs sont systématiquement supérieurs chez les hommes: dans l'ensemble, les hommes consomment $65,0 \mathrm{~g} / \mathrm{j}$ de lipides d'origine ani-

male (soit $65 \%$ ) et les femmes $48,6 \mathrm{~g} / \mathrm{j}$ (soit $61,2 \%)$. L'apport brut en lipides d'origine animale tend à diminuer dans la classe d'âge 5579 ans, aussi bien chez les hommes que chez les femmes.

La figure 1 montre les groupes d'aliments contributeurs aux apports en lipides d'origine animale chez les filles, les garçons, les femmes et les hommes. Quels que soient le sexe et l'âge, le beurre est le principal vecteur de lipides animaux : il contribue à $28 \%$ de la ration des hommes, à $30 \%$ de celle des garçons et à $32 \%$ de celle des filles et des femmes. Le deuxième groupe vecteur est celui des fromages qui apportent 16 et $19 \%$ des apports en lipides d'origine animale des hommes et des femmes et $13 \%$ de ceux des enfants. Chez les garçons, ce groupe partage sa deuxième place avec la viande de porc (yc jambon et charcuteries). Chez les filles et les adultes, la viande de porc est le troisième vecteur et contribue à respectivement 11,12 et $16 \%$ des apports des filles, des femmes et des hommes. Le lait apporte $11 \%$ des lipides animaux des enfants, ce qui en fait le quatrième vecteur, alors que chez l'adulte sa contribution est diminuée de moitié, ce qui en fait une source moins importante que la viande de bœuf et équivalente aux aliments de type yaourts, fromages blancs, etc. Globalement, le lait et les produits dérivés du lait apportent entre $66 \%$ des lipides animaux (chez les filles) et $60 \%$ (chez les hommes). Les viandes, quant à elles, contribuent entre $30 \%$ (chez les garçons) et $27 \%$ (chez les filles) des apports. Les œufs apportent en moyenne entre 4 et $5 \%$ des lipides animaux et les produits de la mer (poissons, coquillages, crustacés) entre $1 \%$ (chez les enfants) et $3 \%$ (chez les femmes).

S'il n'était pas possible de comparer les apports en lipides $d^{\prime}$ origine exclusivement animale entre INCA1 et INCA2, il était possible de comparer les apports d'origine animale ou mixte (incluant par exemple les apports lipidiques totaux des pâtisseries, des tartes salées, etc. et autres préparations à base de lipides animaux et végétaux). Par comparaison avec les apports en lipides $d$ 'origine exclusivement animale (tableau 1), ce groupe mixte contient en moyenne entre 8 et $9 \mathrm{~g}$ de lipides supplémentaires. La figure 2 montre qu'entre les deux études, les apports en lipides animaux ou mixtes ont baissé en moyenne de $14 \%$, de manière un peu plus marquée chez les femmes et les filles (17 \%) que chez les hommes et les garçons (12 et $13 \%)$.

\section{Discussion}

Les analyses effectuées présentent certaines limites. Tout d'abord, comme dans toute enquête alimentaire, même si les personnes 


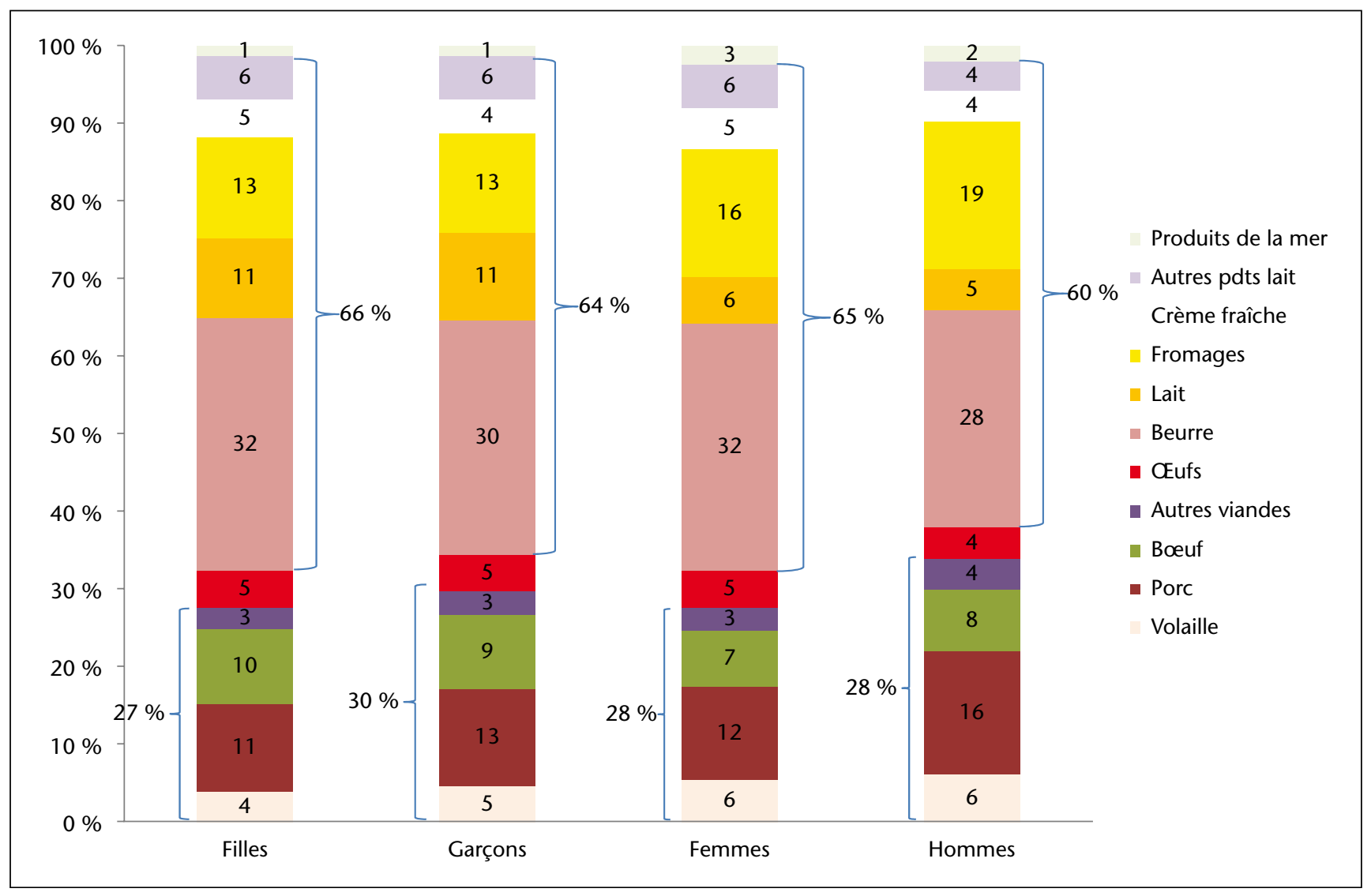

Figure 1. Groupes d'aliments contributeurs aux apports en lipides d'origine animale, chez les filles et les garçons de 3-17 ans et chez les hommes et les femmes de 18-79 ans de l'étude INCA2 (2006-2007).

dont les apports caloriques sont sous-évalués de manière quasi certaine sont exclues de l'analyse, il reste très probablement une part de sous-estimation non identifiée. Celle-ci peut concerner certains aliments de manière différentielle, notamment ceux riches en graisses. Ensuite, comme dans toute enquête alimentaire par enregistrement, on ne peut exclure une modification du comportement des participants pendant l'étude. Enfin, des erreurs de saisie et de codification des aliments sont possibles. En lien avec la décomposition des plats composés et des aliments complexes en ingrédients, il n'était pas toujours possible de distinguer les différences possibles dans leur préparation ou leur origine. Dans la majorité des cas, une recette moyenne a été utilisée.

L'étude INCA2 est cependant la première étude nationale à présenter les apports lipidiques selon leur origine. Les lipides d'origine animale représentent en moyenne entre 59 et $66 \%$ des apports lipidiques totaux selon le sexe et la classe d'âge. Dans le rapport de l'étude d'imprégnation par les dioxines des populations vivant à proximité d'usines d'incinération d'ordures ménagères (InVS, 2009), les apports en lipides d'origine animale de 1030 personnes âgées de 30 à 65 ans ont été estimés à $68 \mathrm{~g} / \mathrm{j}$, soit $64 \%$ de l'apport lipidique total. Bien que cette population soit très différente de celle de l'étude INCA2, avec notamment une sur-représentation des personnes vivant en milieu semi-rural, pratiquant l'autoconsommation, et une méthode d'enquête différente (questionnaire de fréquence), les estimations sont très proches.

Cette estimation peut également être rapprochée de celle effectuée sur la cohorte de femmes françaises de l'étude EPIC pour les apports protéiques, montrant que $66 \%$ de ces apports proviennent d'aliments animaux (Halkjær et al., 2009).

Une comparaison des apports lipidiques d'origine animale n'était pas possible entre INCA1 et INCA2 pour plusieurs raisons : la décomposition des recettes lors de l'étude INCA1 n'avait pas été aussi fine que celle réalisée lors d'INCA2, d'où une part d'incertitude sur l'origine des lipides. De plus, lors d'INCA2, la consommation des matières grasses ajoutées lors de la préparation des plats ou lors de leur service a été améliorée par l'utilisation d'un questionnaire ne permettant pas de comparer les consommations de matières grasses ajou- tées entre les deux études. L'évolution des apports lipidiques d'origine animale ou mixte montre une baisse en moyenne de $14 \%$, un peu plus marquée dans la population féminine (17\%). L'évolution des consommations des groupes de produits animaux montre également des baisses. En effet, la consommation de lait a diminué de $24 \%$ chez les adultes entre INCA1 et INCA2, celle des fromages de $14 \%$, celle des viandes de type bœuf, veau, porc, mouton de $10 \%$ (Afssa, 2009 ; Dubuisson et al., 2009). Ces baisses ont été plus marquées chez les femmes: $-31 \%$ pour le lait, $-18 \%$ pour les fromages et les viandes. Chez les enfants de 3 à 14 ans, les consommations de lait, de fromages et de viandes (bœuf, veau, porc, mouton) ont également baissé entre 14 et $19 \%$ (Afssa, 2009 ; Lioret et al., 2009).

Ces baisses de consommation observées sont plus marquées que celles observées sur les lipides d'origine animale ou mixte pour deux raisons. Tout d'abord, ce groupe contient environ 10 à $12 \%$ de graisses d'origine végétale. Ensuite, d'autres groupes d'aliments susceptibles de contenir des graisses d'origine animale ont vu leur consommation augmenter: la consommation des glaces et desserts glacés a 


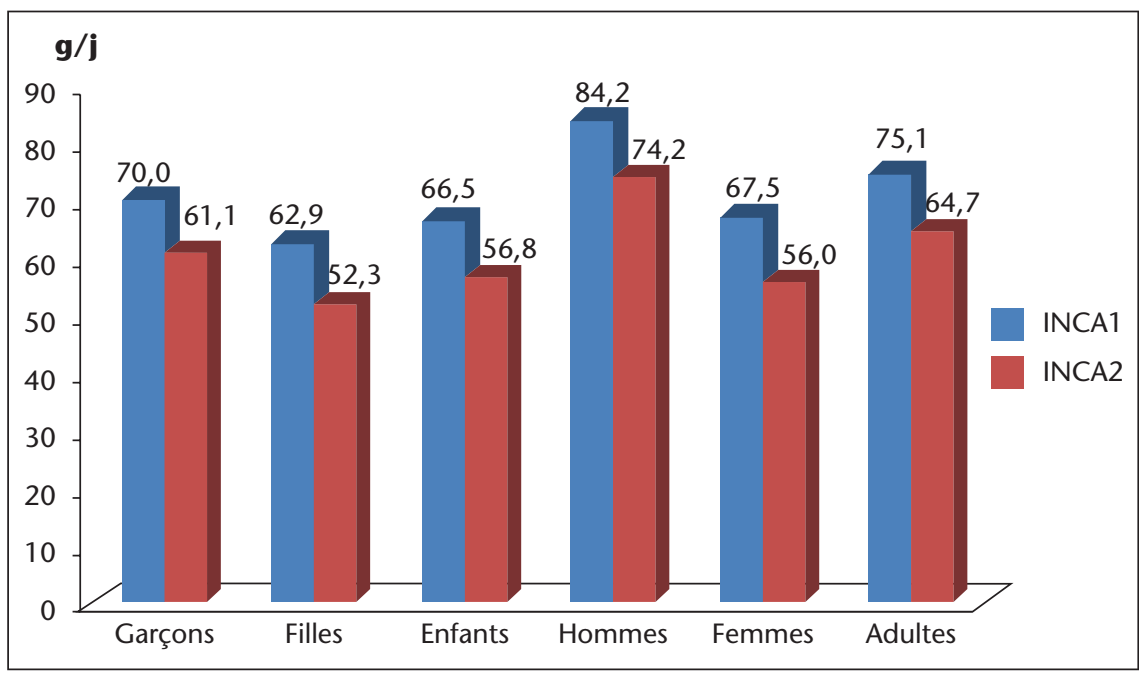

Figure 2. Évolution des apports en lipides animaux et mixtes entre INCA1 (1998-1999) et INCA2 (2006-2007).

notamment augmenté de $21 \%$ chez les enfants de 3-14 ans, de $30 \%$ chez ceux de $15-17$ ans et de $32 \%$ chez les adultes; celle des chocolats et barres chocolatées a augmenté de $54 \%$ chez les 15 à 17 ans, et de $58 \%$ chez les adultes (Afssa, 2009).

L'étude INCA2 a permis d'évaluer la part des lipides d'origine animale dans l'alimentation de la population française. Ces informations seront utiles à la fois d'un point de vue de la sécurité sanitaire en lien avec les substances toxiques véhiculées par les graisses (dioxines ou PCB par exemple), mais également d'un point de vue nutritionnel afin d'évaluer les stratégies d'amélioration des apports en certains nutriments, par exemple en acide oméga-3.

\section{RÉFÉRENCES}

Afssa. Rapport de l'étude individuelle nationale des consommations alimentaires 2 (INCA2). Coord: L Lafay. Février 2009. http://www.afssa.fr/Documents/PASER-Ra-INCA2.pdf.

Afssa. Table de composition nutritionnelle des aliments. Ciqual, 2008. URL: http://www.afssa.fr/TableClQUAL/.

Astorg P, Arnault N, Czernichow S, Noisette N, Galan $P$, Hercberg S. Dietary intakes and food sources of $n-6$ and n-3 PUFA in French adult men and women. Lipids $2004 ; 39: 527-35$.

Black AE. Critical evaluation of energy intake using the Goldberg cut-off for energy intake: basal metabolic rate. A practical guide to its calculation, use and limitations. Int / Obes Relat Metab Disord 2000 ; 24 : 1119-30.
Dubuisson C, Lioret S, Touvier M, et al. Trends in food and nutritional intakes of French adults from 1999 to 2007: results from the INCA surveys. $\mathrm{Br}$ I Nutr 2009; Forthcoming: 1-14. [Epub ahead of print].

Féart C, Jutand MA, Larrieu S, et al. Energy, macronutrient and fatty acid intake of French elderly community dwellers and association with sociodemographic characteristics: data from the Bordeaux sample of the Three-City Study. Br / Nutr 2007 ; 98 : 1046-57.

Goldberg GR, Black AE, Jebb SA, et al. Critical evaluation of energy intake data using fundamental principles of energy physiology: 1 . Derivation of cut-off limits to identify under-recording. Eur / Clin Nutr 1991 ; 45 : 569-81.

Halkjær J, Olsen A, Bjerregaard LJ, et al. Intake of total, animal and plant proteins, and their food sources in 10 countries in the European Prospective Investigation into Cancer and Nutrition. Eur / Clin Nutr $2009 ; 63:$ : $16-36$.

InVS. Étude d'imprégnation par les dioxines des populations vivant à proximité d'usines d'incinération d'ordures ménagères. Rapport d'étude. 2009. http://www. invs.sante.fr/publications/2009/impregnation_dioxines_uiom/index.html.

Lioret S, Dubuisson C, Dufour A, et al. Trends in food intake in French children from 1999 to 2007: results from the INCA (étude individuelle nationale des consommations alimentaires) dietary surveys. $\mathrm{Br}$ I Nutr 2009; Forthcoming:1-17 [Epub ahead of print].

Razanamahefa L, Lafay L, Oseredczuk M, et al. Dietary fat consumption of the French population and quality of the data on the composition of the major food groups. Bull Cancer $2005 ; 92$ : 647-57. 